\title{
Identification of IncRNAs by microarray analysis reveals the potential role of IncRNAs in cervical cancer pathogenesis
}

\author{
JIAMING HUANG ${ }^{*}$, TIANYU LIU* , CHUNLIANG SHANG, YUNHE ZHAO, \\ WEI WANG, YANCHUN LIANG, LUYAN GUO and SHUZHONG YAO \\ Department of Obstetrics and Gynecology, The First Affiliated Hospital, \\ Sun Yat-sen University, Guangzhou, Guangdong 510800, P.R. China
}

Received February 6, 2016; Accepted April 24, 2017

DOI: $10.3892 / \mathrm{ol} .2018 .8037$

\begin{abstract}
Long non-coding RNAs (lncRNAs) have been acknowledged to serve a significant role in cancer biology and abnormal expression in tumors is frequently observed. However, their mechanisms in cervical cancer remain unclear. With a genome-wide analysis of IncRNA expression in cervical cancer tissues, the present study aimed to identify lncRNA targets for the further study of cervical cancer. To elucidate the specific role of IncRNAs in the pathogenesis of this type of cancer, 6 cervical cancer samples paired with normal cervical tissues were obtained. Expression profiles of lncRNAs and mRNAs were constructed through microarray analysis and confirmed by reverse transcription-quantitative polymerase chain reaction (RT-qPCR) methods. Gene Ontology and pathway enrichment analyses were performed with computational methods. On the basis of correlations between the differential expression levels of lncRNAs and mRNAs, a coding-non-coding gene co-expression network (CNC network) was established. The differential expression of 5,844 lncRNAs and 4,436 mRNAs were discovered in cervical cancer samples compared with normal cervical tissues. Among the differentially expressed lncRNAs, 14 were chosen at random and validated by RT-qPCR; the majority of the results measured were consistent with the microarray results. Furthermore, the lncRNA ENST00000551152 was found to be upregulated and TCO. NS_00001368 lncRNA was downregulated in cervical cancer cell lines. The CNC network included 592 network nodes and 934 associations between 12 lncRNAs and 580 protein-coding genes, indicating that one lncRNA could act on a maximum of 141 coding genes, and that one coding gene may corresponded with a maximum of 5 lncRNAs.
\end{abstract}

Correspondence to: $\mathrm{Dr}$ Shuzhong Yao, Department of Obstetrics and Gynecology, The First Affiliated Hospital, Sun Yat-sen University, 58 Zhongshan Second Road, Guangzhou, Guangdong 510800, P.R. China

E-mail: yszlfy@163.com

*Contributed equally

Key words: lncRNA, microarray, cervical cancer
Overall, the present study has provided a complete expression profile of lncRNAs and mRNAs in cervical cancer, which may now be used to establish a solid foundation for cervical cancer research. These results may provide significant information for improving the understanding of the pathogenesis of cervical cancer and indicate potential therapeutic targets.

\section{Introduction}

Cervical cancer is the most common type of malignant neoplasm in the female reproductive system and ranks as the second most common cause of gynecological cancer-associated mortality, with 47,130 new cases and 8,010 mortalities reported in the United States in 2014 (1). Despite decreases in the incidence and mortality rates of cervical cancer, which are largely due to progress in surgical treatment (2) radiotherapy (3) and chemotherapy (4), cervical cancer remains a substantial threat to women's health globally (5). It has been hypothesized that the carcinogenesis and development of cervical cancer are associated with sexual behavior, childbirth, persistent human papillomavirus (HPV) infection and smoking (6). Extensive efforts have been made to clarify the genetic and epigenetic mechanisms that underlie the growth and invasion of cervical cancer. However, the pathogenesis remains understood. Pelvic lymph node metastasis may serve as an independent prognostic factor (7); however, the lack of specific tumor markers for the prediction of cervical cancer mortality and invasion has yet to be resolved.

Long non-coding RNA (lncRNA) is defined as RNA of $>200$ nucleotides without protein-coding function. It has been reported that the portion of the human genome that encodes proteins may be $<2 \%$ (8). A growing number of lncRNAs have been recognized as key regulators, rather than simply transcriptional noise. They are associated with various biological processes, including genomic imprinting, $\mathrm{X}$ chromosome inactivation, chromatin modification, transcription interference, transcription activation and nuclear transport (8-10). lncRNAs are one of the most highly expressed classes of non-coding RNAs in human cervical tissue, and their relevance to cervical cancer is steadily becoming more apparent (11). A previous study demonstrated that lncRNA expression levels differ, with statistical significance, between three cervical intraepithelial neoplasia grades, indicating that these transcripts may be 
involved in the development and progression of pre-cancerous lesions (12). Studies on this topic have revealed that aberrant expression of certain lncRNAs, including HOX transcript antisense RNA (HOTAIR), metastasis-associated lung adenocarcinoma transcript 1 , cervical carcinoma-expressed PCNA regulatory lncRNA, IncRNA-EBIC (also known as thymopoietin pseudogene 2), growth arrest-specific 5, and lncRNA-LET (also known as NPTN intronic transcript 1), serves critical roles in cervical cancer development, invasion and metastasis (13-18). However, none of these lncRNAs has been applied as biomarker for clinical diagnosis. Furthermore, the precise function of the majority of lncRNAs remains unknown despite extensive research efforts. Thus, there is an urgent requirement to identify the mechanisms of interaction between lncRNAs and coding genes.

In the present study, a high-throughput microarray was employed to analyze lncRNA and mRNA expression profiles in samples of cancerous and normal cervical tissue. Gene Ontology (GO) and pathway enrichment analyses investigated enriched functions of the predicted targets, allowing the establishment of an IncRNA-mRNA correlation network. The results illustrated that differences in the expression levels of IncRNAs, in addition to mRNAs, may be associated with cervical cancer pathogenesis; the comprehensive analysis of lncRNAs and mRNA may lay a foundation for further investigation in the diagnosis and treatment of cervical cancer.

\section{Materials and methods}

Patient specimens and RNA extraction. A total of 6 cervical carcinoma samples and 6 normal cervical tissues were collected for microarray. The 6 cervical carcinoma samples were labeled $\mathrm{C} 1$ and C3-7, C2 was diagnosed cervical carcinoma in situ and was not suitable for microarray. For validation, another 20 normal and 30 cancerous cervical tissue samples were also selected. The number of cervical cancer tissues was subsequently increased to 60 for the study of the association between ENST00000551152 expression and clinicopathological parameters. Patient characteristics are summarized in Table I. All the tissue samples were obtained between January 2014 to January 2015 from the Department of Gynecology, First Affiliated Hospital of Sun Yat-sen University (Guangzhou, China). Samples were selected based on the diagnosis of cervical cancer, determined by at least two pathologists and no patients had received chemotherapy or radiotherapy prior to surgery. All provided written, informed consent. The present study was approved by the Medical Ethics Committees at the First Affiliated Hospital of Sun Yat-sen University. All samples were stored at $-80^{\circ} \mathrm{C}$ until RNA extraction.

Extraction of total RNA from fresh tissues was performed using RNAiso Plus reagent (Takara Biotechnology Co., Ltd., Dalian, China) and the total RNA content of each sample was quantified using a NanoDrop ND-1000 (Thermo Fisher Scientific, Inc., Wilmington, DE, USA). Standard denaturing agarose gel electrophoresis was performed to evaluate the RNA integrity.

RNA labeling and array hybridization. Following the Agilent One-Color Microarray-Based Gene Expression
Analysis protocol (Agilent Technologies, Inc., Santa Clara, CA, USA), sample labeling and array hybridization were implemented in the 6 cancer tissues and 6 normal cervical tissues. Ribosomal RNA was removed to leave purified mRNA using an mRNA-ONLY ${ }^{\mathrm{TM}}$ Eukaryotic mRNA Isolation Kit (Epicentre; Illumina, Inc., San Diego, CA, USA). The entire lengths of the transcripts were transcribed into fluorescent complementary RNA (cRNA) without $3^{\prime}$ bias by using random priming. The labeled cRNA was then purified with an RNeasy Mini Kit (Qiagen China Co., Ltd, Shanghai, China). A mixture containing $5 \mu$ l 10X blocking agent, $1 \mu \mathrm{g} 25 \mathrm{X}$ fragmentation buffer and $1 \mu \mathrm{g}$ of each labeled cRNA sample was fragmented and then heated for $30 \mathrm{~min}$ at $60^{\circ} \mathrm{C}$. To dilute the labeled cRNA, $25 \mu 12 \mathrm{X} \mathrm{GE}$ Hybridization buffer (GE Healthcare Life Sciences, Logan, UT, USA) was added. After dispensing into the gasket slide, $50 \mu \mathrm{l}$ of hybridization solution was applied to the lncRNA expression microarray slide. Incubation of the slides was performed in an Agilent hybridization oven at $65^{\circ} \mathrm{C}$ for $17 \mathrm{~h}$ (Agilent Technologies, Inc.). Washing, fixing and scanning of the hybridized arrays were completed with the Agilent DNA microarray scanner (part no. G25005C).

Data analysis of IncRNA and mRNA expression profiles. The general profiles of human lncRNAs and protein-coding transcripts from the 6 cervical carcinoma samples and 6 normal cervical tissues were detected using the Arraystar Human lncRNA Microarray V3.0 (Arraystar, Inc., Rockville, MD, USA); using this array, 26,109 coding transcripts and 30,586 lncRNAs are detectable. Array images were analyzed by Agilent Feature Extraction software (version 11.0.1.1; Agilent Technologies, Inc.). Following quantile normalization and data processing in GeneSpring GX v11.5.1 software package (Agilent Technologies, Inc.), lncRNA and mRNAs flagged as Present or Marginal ('all Target Value') in $\geq 6$ out of 12 samples and were selected for further analysis. Volcano plot filtering was employed to validate the significance of the differences in the IncRNA and mRNA expression levels between the cancer and normal cervical tissues. GeneSpring GX was used to perform hierarchical clustering. GO and pathway analyses were performed using standard enrichment computation.

Reverse transcription-quantitative polymerase chain reaction $(R T-q P C R)$. Following total RNA extraction as described, cDNA synthesis was performed for $15 \mathrm{~min}$ at $37^{\circ} \mathrm{C}$ and $5 \mathrm{sec}$ at $85^{\circ} \mathrm{C}$ with PrimeScript RT Master Mix (Takara Biotechnology Co., Ltd.) according to the manufacturer's protocol. Primer sequences are listed in Table II. SYBR Premix Ex Taq (Takara Biotechnology Co., Ltd.) was used with $2 \mu 1$ template cDNA in each reaction for qPCR with a 7500 Fast Real-Time PCR System (Applied Biosystems; Thermo Fisher Scientific, Inc.). In the 40 cycles of PCR, pre-denaturation was performed for $30 \mathrm{sec}$ at $95^{\circ} \mathrm{C}$, while the parameters for denaturation and annealing were set at $95^{\circ} \mathrm{C}$ for $5 \mathrm{sec}$ and $60^{\circ} \mathrm{C}$ for $34 \mathrm{sec}$, respectively. The $2^{-\Delta \Delta \mathrm{Cq}}$ value $[\Delta \mathrm{Cq}=\mathrm{Cq}(\mathrm{RNA})-\mathrm{Cq}(\mathrm{GAPDH})](19)$ was used to determine an average cycle threshold value from triplicate reactions and calculate the level of genomic expression (20). Single product amplification was ensured by the generated melting curves. 
Table I. Correlation between ENST00000551152 expression and clinicopathological characteristics in early-stage squamous cervical cancer.

\begin{tabular}{|c|c|c|c|c|}
\hline \multirow[b]{2}{*}{ Characteristic } & \multirow[b]{2}{*}{ All patients, $\mathrm{n}$} & \multicolumn{2}{|c|}{ ENST00000551152 expression, $\mathrm{n}(\%)$} & \multirow[b]{2}{*}{ P-value } \\
\hline & & Low & High & \\
\hline Total & 60 & $14(23.3)$ & $46(76.7)$ & \\
\hline Age, years & & & & 0.297 \\
\hline$\leq 42$ & 33 & $6(18.2)$ & $27(81.8)$ & \\
\hline$>42$ & 27 & $8(29.6)$ & $19(70.4)$ & \\
\hline FIGO stage & & & & $0.017^{\mathrm{a}}$ \\
\hline IB1 & 29 & $12(41.4)$ & $17(58.6)$ & \\
\hline IB2 & 8 & $1(12.5)$ & $7(87.5)$ & \\
\hline IIA1 & 17 & $1(5.9)$ & $16(94.1)$ & \\
\hline IIA2 & 6 & $0(0.0)$ & $6(100.0)$ & \\
\hline Tumor size, $\mathrm{cm}$ & & & & $0.022^{\mathrm{a}}$ \\
\hline$\leq 4$ & 39 & $13(33.3)$ & $26(66.7)$ & \\
\hline$>4$ & 21 & $1(4.8)$ & $20(95.2)$ & \\
\hline Differentiation grade & & & & 0.056 \\
\hline Well-differentiated & 7 & $4(57.1)$ & $3(42.9)$ & \\
\hline Moderately differentiated & 25 & $6(24.0)$ & $19(76.0)$ & \\
\hline Poorly differentiated & 28 & $4(14.3)$ & $24(85.7)$ & \\
\hline Extent of stromal invasion & & & & 0.001 \\
\hline$<1 / 2$ & 29 & $12(41.4)$ & $17(58.6)$ & \\
\hline$\geq 1 / 2$ & 31 & $2(6.5)$ & $29(93.5)$ & \\
\hline LVSI & & & & $0.053^{\mathrm{a}}$ \\
\hline Yes & 11 & $0(0.0)$ & $11(100.0)$ & \\
\hline No & 49 & $14(28.6)$ & $35(71.4)$ & \\
\hline PLNM & & & & $0.026^{\mathrm{a}}$ \\
\hline Yes & 14 & $0(0.0)$ & $14(100.0)$ & \\
\hline No & 46 & $14(30.4)$ & $32(69.6)$ & \\
\hline SCC antigen, ng/ml & & & & 0.004 \\
\hline$<1.5$ & 27 & $11(40.7)$ & $16(59.3)$ & \\
\hline$\geq 1.5$ & 33 & $3(9.1)$ & $30(90.9)$ & \\
\hline
\end{tabular}

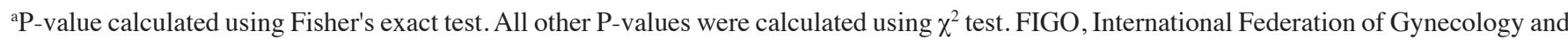
Obstetrics; LVSI, lymphovascular space invasion; PLNM, pelvic lymph node metastasis; SCC, squamous cell carcinoma.

GO and pathway analysis. GO is a functional analysis that annotates genes and attributes associated with their expression using ontological categories, including 'biological process', 'cellular component' and 'molecular function'. GO categories (http://www.geneontology.org) were applied to the differentially expressed lncRNAs (21). To analyze how the target genes function in the cellular pathways, the Kyoto Encyclopedia of Genes and Genomes (KEGG; http://www.kegg.jp) (22) database was used. The P-value, which represents the importance of the pathway, was used with a cut-off 0.5 , with lower values indicating greater significance.

lncRNA-mRNA correlation network. The lncRNA-mRNA correlation network was established according to lncRNA target predictions along with differentially expressed lncRNA and mRNA profiles using Cytoscape (http://www. cytoscape.org) (23). The lncRNAs and mRNAs that were selected to create the network were those with Pearson correlation coefficients $\geq 0.99$.

Cell culture. The cervical carcinoma cell lines HeLa, SiHa, MS751 and C33A were purchased from the American Type Culture Collection (Manassas, VA, USA) and cultured in Dulbecco's Modified Eagle's medium (Gibco; Thermo Fisher Scientific, Inc.) or RPMI-1640 medium (BRI, Rockville, MD, USA), supplemented with $10 \%$ fetal bovine serum (Hyclone; GE Healthcare Life Sciences), penicillin (100 U/ml), and streptomycin $(100 \mu \mathrm{g} / \mathrm{ml})$. The conditions during cell culture were $5 \% \mathrm{CO}_{2}, 95 \%$ humidified air, and $37^{\circ} \mathrm{C}$.

Statistical analysis. Statistical analyses were performed using SPSS software (version 13.0; SPSS, Inc., Chicago, 
Table II. Primers used for quantitative polymerase chain reaction analysis of long non-coding RNA levels.

\begin{tabular}{|c|c|}
\hline Sequence ID & Primer sequence ( $5^{\prime}$ to $3^{\prime}$ ) \\
\hline \multirow[t]{2}{*}{ TCONS_00001368 } & F: CACACAAGGACTGGAGCAAA \\
\hline & R: CACCTAАCCCACCACATTCA \\
\hline \multirow[t]{2}{*}{ NR_033746 } & F: GCAGCTCAGGTTCTCCAAAT \\
\hline & R: CCCTCTTTAGCCTGTTGGTC \\
\hline \multirow[t]{2}{*}{ NR_027122 } & F: CTGTCCTCCTGCTCTTTGC \\
\hline & R: GAGTTTTGGGTTCACGGATA \\
\hline \multirow[t]{2}{*}{ ENST00000421943 } & F: GGGACCAGGAATGTGAACTT \\
\hline & R: TGCCCTCAGATGTGAAACTC \\
\hline \multirow[t]{2}{*}{ ENST00000439076 } & F: ACAGGCGGCAGAGAAGAAG \\
\hline & R: GACACACGCAGTCATTCAGG \\
\hline \multirow[t]{2}{*}{ ENST00000448991 } & F: GCAGACTTGACCTCTTGGC \\
\hline & R: ATAGTGGGTATCGGGGGTG \\
\hline \multirow[t]{3}{*}{ uc001iot.1 } & F: GAGAAGAGGCGAACGAGG \\
\hline & R: GTGGGACAGCCAATACATA \\
\hline & AT \\
\hline \multirow[t]{2}{*}{ ENST00000414085 } & F: CGCAGAACTTTGCTGGAGA \\
\hline & $\begin{array}{l}\text { R: GAAATACAGAGTCAGAGAGC } \\
\text { GTG }\end{array}$ \\
\hline \multirow[t]{3}{*}{ ENST00000421498 } & F: GACCATGCTGTTGAAACCAC \\
\hline & R: TCAAGGAGAGCACAAGGAA \\
\hline & $\mathrm{CT}$ \\
\hline \multirow[t]{2}{*}{ ENST00000428667 } & F: TTTCCATACCCAGCCAACTT \\
\hline & R: CTTCCTGCACTGCCAACCT \\
\hline \multirow[t]{2}{*}{ ENST00000443523 } & F: CCTGGCTGGAGATGCTTACT \\
\hline & R:GGTTCCTGTTGGGACTTTAGA \\
\hline \multirow[t]{2}{*}{ ENST00000551152 } & F: GCAAGAACTGAGACCTGACG \\
\hline & R: TAAGCACACCACTCCACTGC \\
\hline \multirow[t]{2}{*}{ GAPDH } & F: GGGAAACTGTGGCGTGAT \\
\hline & R: GAGTGGGTGTCGCTGTTGA \\
\hline
\end{tabular}

F, forward; R, reverse.

IL, USA). All data are presented as the mean \pm standard deviation. The Student's t-test was used for evaluating the statistical significance of differences in the means between two groups. The association between lncRNA expression and clinicopathological features was assessed using the $\chi^{2}$ test and Fisher's exact test. $\mathrm{P}<0.05$ was considered to indicate a statistically significant difference. Receiver operating characteristic (ROC) curve analysis was employed to define the cutoff value for high expression of lncRNA by the $(0,1)$-criterion, and the area under the curve was calculated as previously described (24).

\section{Results}

Aberrant lncRNA and mRNA expression in cervical cancer. The profiles of IncRNAs and mRNAs in paired cervical cancer and normal cervical tissue samples were produced using microarray technology to investigate the potential biological function of lncRNAs in cervical cancer (Fig. 1A and B).
Relative to normal tissue, 5,844 (19.1\%) lncRNAs presented differential expression in cancer tissue (fold-change, $\geq 2$ ) among the 30,586 detected lncRNA transcripts. This included 2,574 upregulated 3,270 downregulated lncRNAs. The most prominently upregulated and downregulated lncRNAs were uc002jcf.3 (fold-change, 57.214) and NR_027122 (fold-change, $620.995)$, respectively.

In the mRNA expression profile data, 1,538 upregulated mRNAs and 2,898 downregulated mRNAs were detected (Fig. 1C and D). Among these mRNAs, the most prominently upregulated and downregulated mRNAs were Kelch domain-containing 7B (fold-change, 224.009) and keratin 1 (fold-change, 339.108), respectively.

Ontological and pathway analysis of target genes of differentially expressed IncRNAs. To investigate a possible correlation between functional grouping and the differentially expressed lncRNAs, GO annotation categories including 'biological process' (Fig. 2A and B), 'cellular component' (Fig. 2C and D) and 'molecular function' (Fig. 2E and F) were explored for the differentially expressed mRNAs of the samples. In the GO 'biological process' classification, the majority of the upregulated and downregulated GO annotations were associated with 'cellular process' and 'regulation of cellular process'. The 'cellular component' classification search revealed that a large proportion of genes that were upregulated in cervical cancer were annotated as GO categories 'intracellular', 'intracellular part', 'organelle' and 'intracellular organelle'; 'cell' and 'cell part' accounted for the greatest numbers of downregulated genes. The most common 'molecular function' GO annotations for all differentially expressed genes were associated with 'binding' and 'protein binding'.

Additionally, pathway analysis was accomplished with the KEGG database (22) to investigate the biological pathways associated with the mRNAs with the most pronounced differential expression in cervical cancer. A total of 40 upregulated pathways were identified in the cervical cancer tissue, which were correlated with the KEGG pathway categories 'cytokine-cytokine receptor interaction', 'chemokine signaling pathway', 'transcriptional misregulation in cancer' and 'cell cycle'. A total of 37 downregulated pathways were identified, which were associated with the categories 'MAPK signaling pathway', 'pathways in cancer' and 'Wnt signal pathway'; all have been previously reported to be associated with cervical cancer (Fig. 3) (25,26).

Construction of lncRNA-mRNA correlation network. In total, 592 network nodes and 934 associations between 12 lncRNAs and 580 coding genes were included in the CNC network, within which there were 500 positive correlation pairs, and 434 negative correlation pairs. The co-expression network demonstrated that one lncRNA could act on 141 coding genes maximally, and that one coding gene corresponded to a maximum of 5 lncRNAs. The mutual regulation between lncRNAs and mRNAs in cervical cancer was also indicated in the CNC network.

$R T$-qPCR validation. To confirm the microarray results, 12 IncRNAs were selected at random and validated by RT-qPCR. The expression levels of these lncRNAs were 

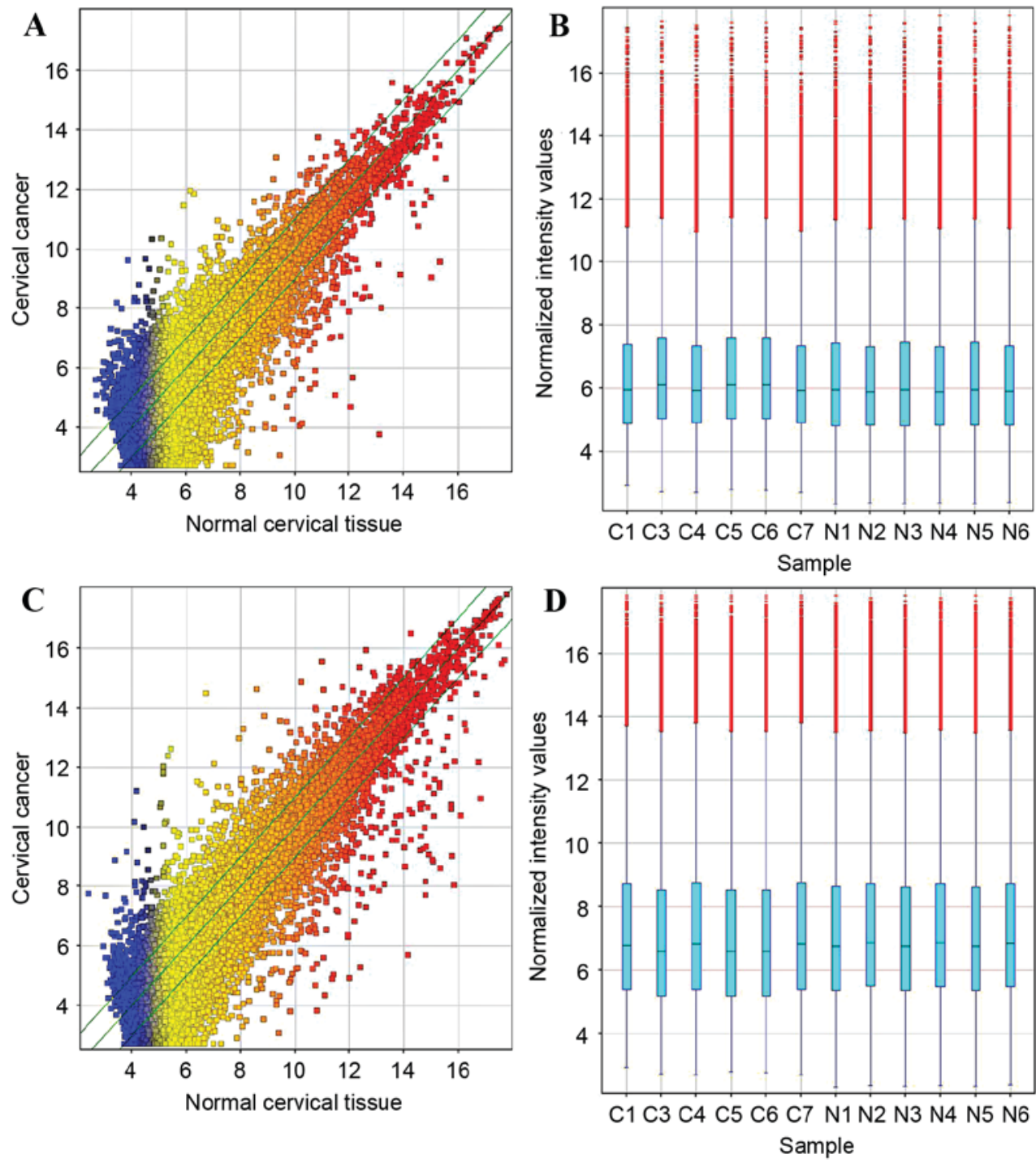

Figure 1. IncRNA and mRNA profiles compared between the 6 cervical cancer samples and 6 normal tissue samples. The scatterplots summarize (A) lncRNA and (C) mRNA expression variation between six cervical cancer samples and six normal cervical samples. The box plots summarize the distribution of (B) lncRNA and (D) mRNA microarray fluorescence intensity values following normalization. IncRNA, long non-coding RNA; C1, C3-7, cervical cancer samples; N1-6, normal cervical tissue samples.

detected in 30 cervical carcinoma tissues and 20 normal cervical tissues. The analysis revealed that the lncRNAs ENST00000414085, ENST00000428667, ENST00000551152 and ENST00000443523 were upregulated, and that ENST00000421943, NR_027122, ENST00000448991, ENST00000439076, TCONS_00001368 and NR_033746 were downregulated in the cervical carcinoma tissues relative to the normal cervical tissues ( $\mathrm{P}<0.05$; Fig. 4 ), in good consistency with the microarray results. However, the lncRNAs ENST00000421498 and uc001iot.1 showed no statistically significant differences in expression between cancerous tissue and normal tissues. In summary, the vast majority of the lncRNAs assessed by RT-qPCR were in line with the trends observed by lncRNA microarray.

For further study on the potential function of lncRNAs, the upregulated lncRNA ENST00000551152 and downregulated lncRNA TCONS_00001368 were investigated in cervical carcinoma cell lines. It was observed that ENST00000551152 was overexpressed in $\mathrm{SiHa}, \mathrm{HeLa}, \mathrm{C} 33 \mathrm{~A}$ and MS751 cervical cancer cell lines compared with normal cervical cancer tissues, whereas the expression of TCONS_00001368 was decreased in these cervical cancer cell lines ( $\mathrm{P}<0.05$; Fig. 5).

Correlation of ENST00000551152 expression and clinicopathological parameters. As ENST00000551152 had the highest level of overexpression among the 12 IncRNAs selected for validation, the association between ENST00000551152 expression level and clinicopathological characteristics in cervical cancer patients was examined to reveal the potential role of this IncRNA in cervical cancer pathogenesis. Additionally, 20 normal and 60 cancerous cervical tissue samples were selected for ENST00000551152 level quantification. The data indicated that the expression of ENST00000551152 in cancer tissues was significantly increased by 2.45 mean fold-change compared with that in normal cervical tissues $(\mathrm{P}<0.05$; Fig. 6).

ROC curve analysis was employed to determine the cutoff value for high ENST00000551152 expression (Fig. 7). 


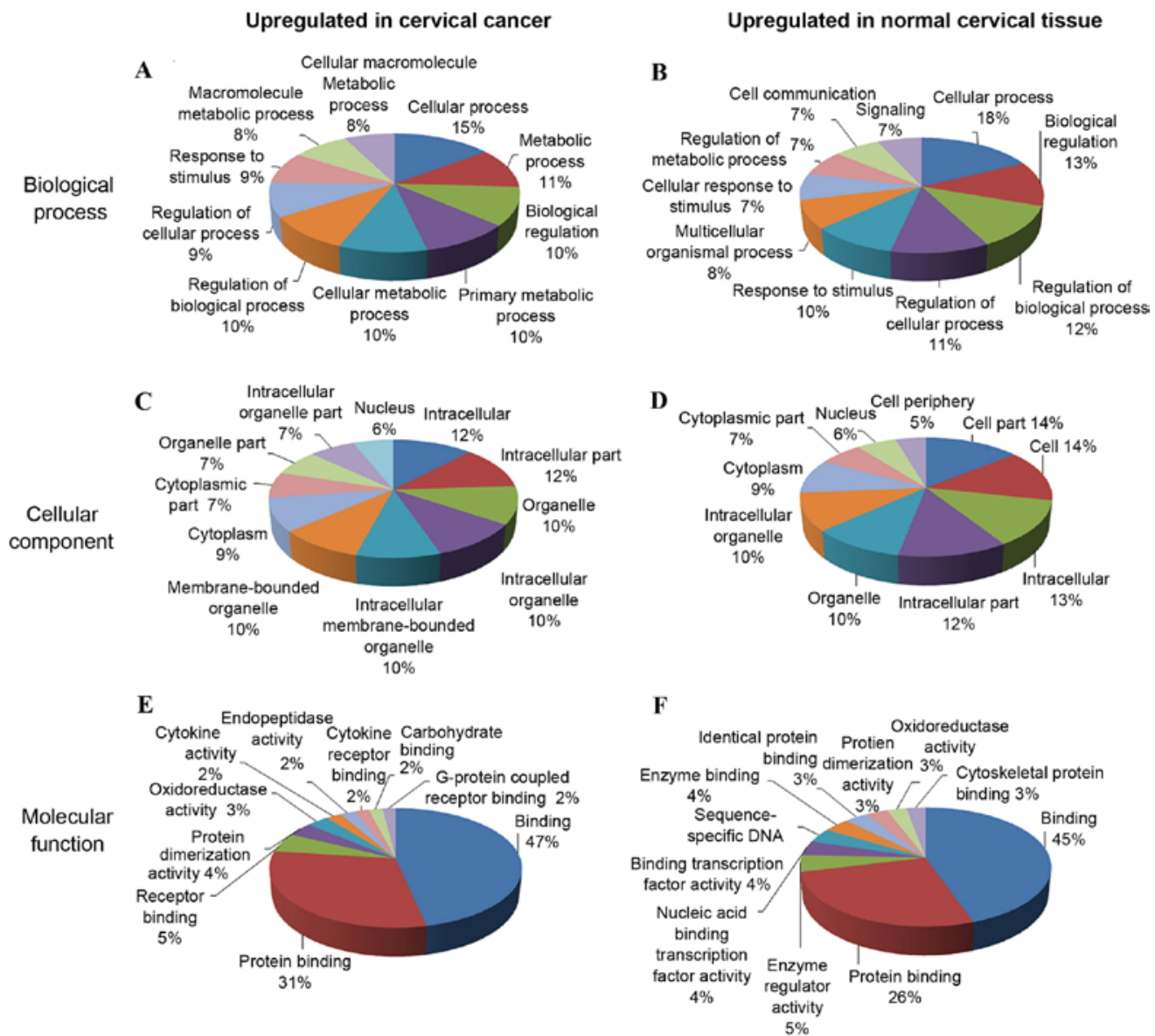

Figure 2. GO analysis of differentially expressed genes. The chart shows the top 10 most significantly enriched GO terms for the different categories. Enriched category annotations in (A and B) 'biological process', (C and D) 'cellular component', and (E and F) 'molecular function' are shown for differentially expressed mRNAs in cervical cancer and normal tissues. GO, gene ontology.

A Upregulated

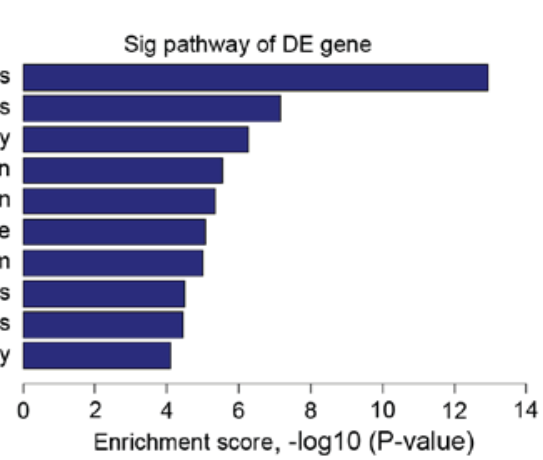

B Downregulated

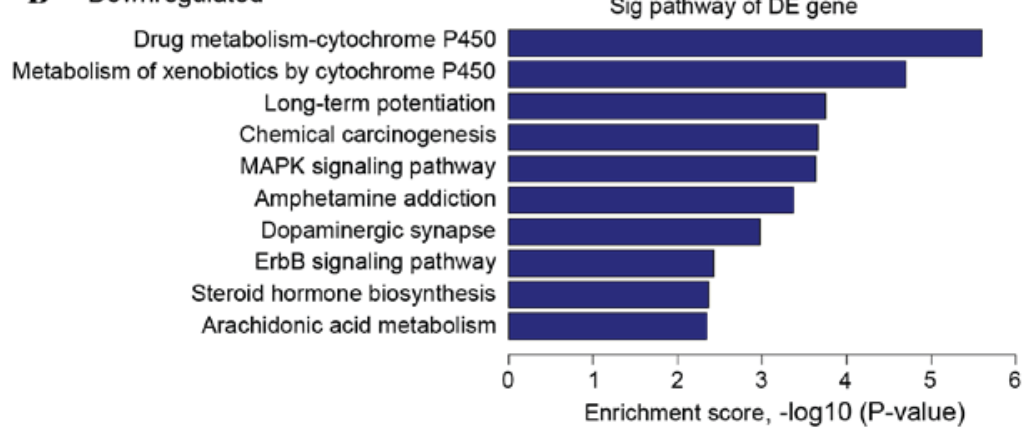

Figure 3. Enriched pathway analysis for DE mRNAs. Pathway analysis was predominantly based on the Kyoto Encyclopedia of Genes and Genomes database. The top 10 pathways associated with (A) upregulated and (B) downregulated DE mRNAs are presented. DE, differentially expressed; sig, significant; MAPK, mitogen-activated protein kinase. 


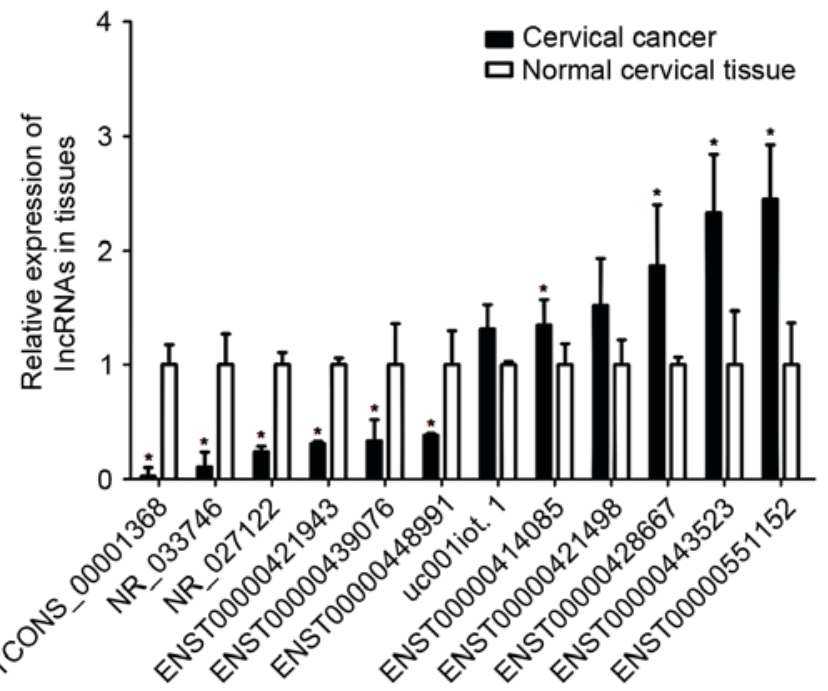

Figure 4. Validation of 12 selected lncRNA expression levels by reverse transcription-quantitative polymerase chain reaction. A total of 30 cervical cancer tissues and 20 normal cervical cancer tissues were used for validation. Of the 12 selected lncRNAs, 10 showed a consistent expression pattern with the microarray data $\left({ }^{*} \mathrm{P}<0.05\right)$. The 2 exceptions to the consistency were IncRNAs ENST00000426615 and uc001iot.1. lncRNA, long non-coding RNA.
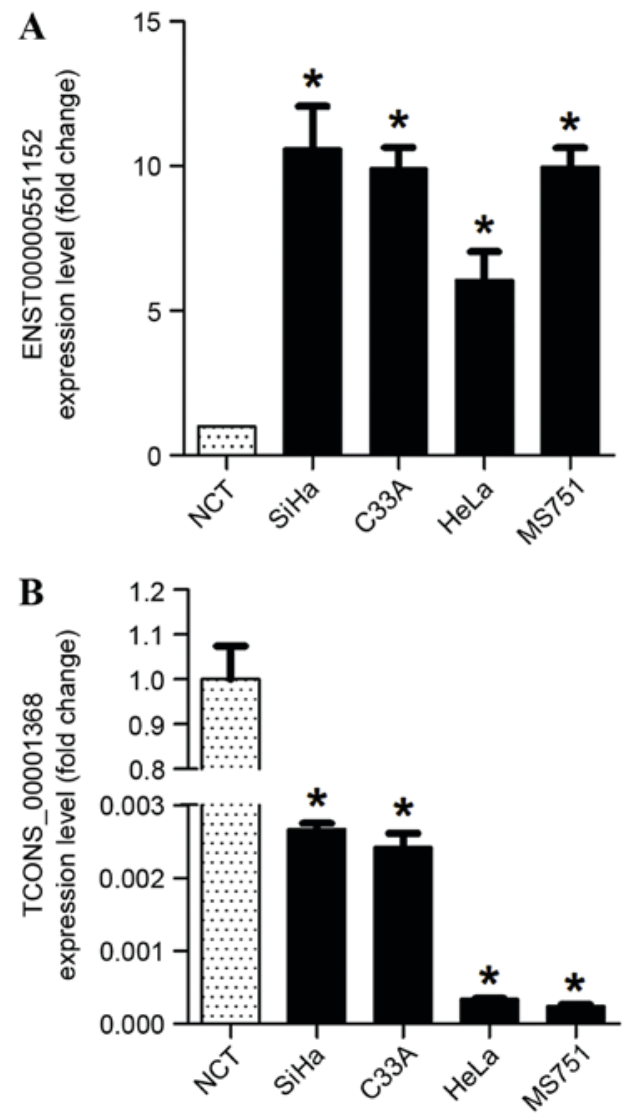

Figure 5. Relative expression levels of two lncRNAs in cervical cancer cell lines. (A) ENST00000551152 lncRNA, which was upregulated in cervical cancer tissue samples, was confirmed to be significantly overexpressed in SiHa, HeLa, C33A and MS751 cervical cancer cell lines when compared with NCT. (B) TCONS_00001368 lncRNA, which was significantly downregulated in cervical cancer tissue samples, was also confirmed to be downregulated in $\mathrm{SiHa}, \mathrm{HeLa}, \mathrm{C} 33 \mathrm{~A}$ and MS751 cervical cancer cell lines when compared with NCT. "P<0.05 vs. NCT. lncRNA, long non-coding RNA; NCT, normal cervical tissues.

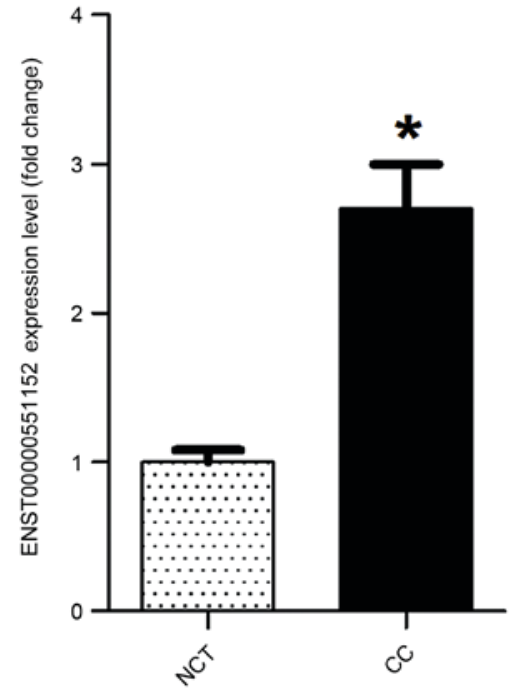

Figure 6. Relative expression of the long non-coding RNA ENST00000551152 in CC tissues. The expression of ENST00000551152 (also known as RP11-396F22) was significantly higher in CC tissues than in NCT. ${ }^{*} \mathrm{P}<0.05$ vs. NCT. CC, cervical cancer; NCT; normal cervical tissues.

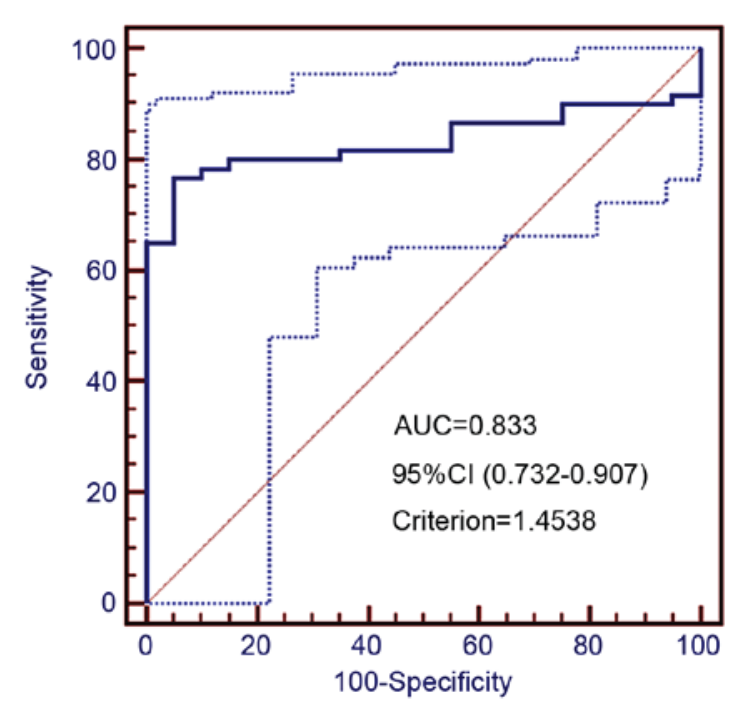

Figure 7. ROC curve analysis. Using a ROC curve analysis, a 1.45-fold increase in the ENST00000551152 long non-coding RNA level in cancer tissues compared with normal tissues was defined as a high level of expression. ROC, receiver operating characteristic; AUC, area under the curve; CI, confidence interval.

Based on this analysis, a total of 46 cancer tissue samples had $\geq 1.45$-fold increased ENST00000551152 levels and were defined as having high level of expression, whereas 14 cases had $<1.45$-fold expression and were considered as having low level expression. Significant correlations were identified between the ENST00000551152 expression level and several prognostic risk factors, including the International Federation of Gynecology and Obstetrics (FIGO) stage $(\mathrm{P}=0.017)$, tumor size $(\mathrm{P}=0.022)$, extent of stromal invasion $(\mathrm{P}=0.001)$, pelvic lymph node metastasis $(\mathrm{P}=0.026)$ and SCC expression level $(\mathrm{P}=0.004)$. No significant difference was found between the expression of ENST00000551152 and other variables, including age, differentiation grade and lymphovascular space invasion (Table I). Taken together, the data indicate that the IncRNA 
ENST00000551152 may serve a critical role in cervical cancer pathogenesis.

\section{Discussion}

The significance of lncRNA in cancer pathogenesis and the correlations between various types of human cancers and the aberrant expression of IncRNAs have been elaborated in numerous studies in the past decade $(27,28)$. Certain lncRNAs behave like oncogenes or tumor-suppressors, performing important functions in cancer initiation, progression, metastasis or recurrence. For example, the lncRNA HOTAIR promotes cellular proliferation, cell cycle progression, migration, and invasion via inhibiting p21 in cervical cancer, and thus functions as an oncogene (17). By contrast, lncRNA-LET serves as a tumor suppressor; the overall survival of patients with cervical cancer with downregulated lncRNA-LET is markedly poorer than in those with lncRNA-LET upregulation (14). Thus, it is hypothesized that lncRNAs may be key regulators in cervical cancer pathogenesis. However, the exact pathogenesis-associated functions of lncRNAs in cervical cancer remain unclear. Thus, it is necessary to identify the expression patterns of lncRNAs on a large scale, which is likely to aid in the identification of novel biomarkers and provide a potential therapeutic target for further research.

In the present study, the differential expression patterns of IncRNAs and mRNAs were profiled by comparing cervical cancer and normal cervical tissue samples to identify the pathogenesis-associated functions of lncRNAs. A total of 5,844 $\mathrm{lncRNAs}$ and 4,436 mRNAs were differentially expressed (with fold-change, $\geq 2$ ) according to the microarray results. Among the 5,844 lncRNAs, 2,574 were upregulated and 3,270 were downregulated, a large portion of which have not yet been functionally characterized. To confirm the consistency of the microarray, 12 lncRNAs were randomly selected for quantification by RT-qPCR in 30 cervical cancer and 20 normal cervical tissue samples. The RT-qPCR results were largely consistent with the microarray data, demonstrating that the high-throughput microarray was able to reflect the actual expression patterns of IncRNAs in cervical cancer tissue samples. In order to gain a better understanding of the biological function of lncRNAs, the expression levels of ENST00000551152 (upregulated) and TCONS_00001368 (downregulated) were assessed in cervical cancer cell lines. Furthermore, the association between the expression of ENST00000551152 and clinicopathological variables were analyzed in tissue samples. It was identified that ENST00000551152 expression levels were closely associated with FIGO stage, tumor size, stromal invasion, pelvic lymph node metastasis and SCC antigen expression level. Thus, ENST00000551152 shows great potential as a biomarker in cervical cancer pathogenesis. Due to the limits of the present study, further study should be conducted to investigate the mechanism by which IncRNAs affect the biology of cervical cancer cells.

Analysis of differentially expressed mRNAs, which were potential targets of the differentially expressed IncRNAs, was completed utilizing the KEGG pathway annotation database in order to increase understanding of the possible functional roles of the lncRNAs. Relative to normal cervical tissues, cervical cancer tissue exhibited upregulated mRNAs that were associated with 40 KEGG pathways. The pathways included 'cytokine-cytokine receptor interaction', 'chemokine signaling pathway', and 'cell cycle', all of which have previously been implicated by a number of studies (29-31). The downregulated mRNAs were enriched for 37 pathways, of which 'MAPK signaling pathway' was the most frequently reported. The CNC network suggested that the regulatory interaction between IncRNAs and mRNAs is complex in cervical cancer. Although elaboration of the exact mechanisms of those genes' involvement in cervical cancer was not achieved, lncRNAs with differential expression may be participants in cervical cancer by regulating these coding genes.

In conclusion, the present study has demonstrated the comprehensive expression profile of IncRNAs and mRNAs in cervical cancer via microarray technology. The differential expression of IncRNAs and mRNAs were observed in cervical cancer samples relative to paired normal cervical tissues. The potential correlation between IncRNAs and protein-coding genes and the roles of IncRNAs in cervical cancer were investigated via bioinformatics analyses, including a $\mathrm{CNC}$ network, KEGG pathway annotation and GO category classification. Detailed regulatory mechanisms remain to be further elaborated. Furthermore, each IncRNA and associated mRNA interaction could be a candidate diagnostic marker or therapeutic target for cervical cancer, and further investigation is required.

\section{Acknowledgements}

The present study was supported by National Natural Science Foundation of China (grant no. 8167100337), the Natural Science Foundation of Guangdong Province (grant no. 2015A030313073) and Science and Technology Program of Guangzhou (grant no. 201510010289).

\section{References}

1. Siegel R, Ma J, Zou Z and Jemal A: Cancer statistics, 2014. CA Cancer J Clin 64: 9-29, 2014.

2. Kucukmetin A, Biliatis I, Ratnavelu N, Patel A, Cameron I, Ralte A and Naik R: Laparoscopic radical trachelectomy is an alternative to laparotomy with improved perioperative outcomes in patients with early-stage cervical cancer. Int J Gynecol Cancer 24: 135-140, 2014.

3. Rijkmans EC, Nout RA, Rutten IH, Ketelaars M, Neelis KJ, Laman MS, Coen VL, Gaarenstroom KN, Kroep JR and Creutzberg CL: Improved survival of patients with cervical cancer treated with image-guided brachytherapy compared with conventional brachytherapy. Gynecol Oncol 135: 231-238, 2014.

4. Tewari KS, Sill MW, Long HJ III, Penson RT, Huang H, Ramondetta LM, Landrum LM, Oaknin A, Reid TJ, Leitao MM, et al: Improved survival with bevacizumab in advanced cervical cancer. N Engl J Med 370: 734-743, 2014.

5. Weinmann S, Williams AE, Kamineni A, Buist DS, Masterson EE, Stout NK, Stark A, Ross TR, Owens CL, Field TS and Doubeni CA: Cervical cancer screening and follow-up in 4 geographically diverse US health care systems, 1998 through 2007. Cancer-Am Cancer Soc 121: 2976-2983, 2015.

6. Koskas M, Rodier JM, Bretel JJ, Bonneau C, Luton D, Touboul C and Rouzier R: Life after uterine cancer. Rev Prat 64: 816-820, 2014.

7. Gien LT and Covens A: Lymph node assessment in cervical cancer: Prognostic and therapeutic implications. J Surg Oncol 99: 242-247, 2009.

8. Caley DP, Pink RC, Trujillano D and Carter DR: Long noncoding RNAs, chromatin and development. ScientificWorldJournal 10: 90-102, 2010. 
9. Lee JT, Davidow LS and Warshawsky D: Tsix, a gene antisense to Xist at the X-inactivation centre. Nat Genet 21: 400-404, 1999.

10. Hung $T$ and Chang HY: Long noncoding RNA in genome regulation: Prospects and mechanisms. RNA Biol 7: 582-585, 2010.

11. Ravasi T, Suzuki H, Pang KC, Katayama S, Furuno M, Okunishi R, Fukuda S, Ru K, Frith MC, Gongora MM, et al: Experimental validation of the regulated expression of large numbers of non-coding RNAs from the mouse genome. Genome Res 16: 11-19, 2006.

12. Gibb EA, Becker-Santos DD, Enfield KS, Guillaud M, Niekerk Dv, Matisic JP, Macaulay CE and Lam WL: Aberrant expression of long noncoding RNAs in cervical intraepithelial neoplasia. Int J Gynecol Cancer 22: 1557-1563, 2012.

13. Cao S, Liu W, Li F, Zhao W and Qin C: Decreased expression of IncRNA GAS5 predicts a poor prognosis in cervical cancer. Int J Clin Exp Pathol 7: 6776-6783, 2014.

14. Jiang S, Wang HL and Yang J: Low expression of long non-coding RNA LET inhibits carcinogenesis of cervical cancer. Int J Clin Exp Pathol 8: 806-811, 2015.

15. Jiang Y, Li Y, Fang S, Jiang B, Qin C, Xie P, Zhou G and Li G: The role of MALAT1 correlates with HPV in cervical cancer. Oncol Lett 7: 2135-2141, 2014.

16. Sun NX, Ye C, Zhao Q, Zhang Q, Xu C, Wang SB, Jin ZJ, Sun SH, Wang $\mathrm{F}$ and $\mathrm{Li}$ W: Long noncoding RNA-EBIC promotes tumor cell invasion by binding to EZH2 and repressing E-cadherin in cervical cancer. PLoS One 9: e100340, 2014.

17. Jing L, Yuan W, Ruofan D, Jinjin Y and Haifeng Q: HOTAIR enhanced aggressive biological behaviors and induced radio-resistance via inhibiting p21 in cervical cancer. Tumour Biol 36: 3611-3619, 2015.

18. Yang M, Zhai X, Xia B, Wang Y and Lou G: Long noncoding RNA CCHE1 promotes cervical cancer cell proliferation via upregulating PCNA. Tumour Biol 36: 7615-7622, 2015.

19. Livak KJ and Schmittgen TD: Analysis of relative gene expression data using real-time quantitative PCR and the 2(-Delta Delta C(T)) method. Methods 25: 402-408, 2001.

20. Sui W, Lin H, Peng W, Huang Y, Chen J, Zhang Y and Dai Y: Molecular dysfunctions in acute rejection after renal transplantation revealed by integrated analysis of transcription factor, microRNA and long noncoding RNA. Genomics 102: 310-322, 2013.

21. Kanehisa M, Goto S, Sato Y, Furumichi M and Tanabe M: Tanabe, KEGG for integration and interpretation of large-scale molecular data sets. Nucleic Acids Res 40: D109-D114, 2012.
22. Gene Ontology Consortium: Gene ontology, gene ontology consortium: Going forward. Nucleic Acids Res 43: D1049-D1056, 2015.

23. Shannon P, Markiel A, Ozier O, Baliga NS, Wang JT, Ramage D, Amin N, Schwikowski B and Ideker T: Cytoscape: A software environment for integrated models of biomolecular interaction networks. Genome Res 13: 2498-2504, 2003.

24. Luo R, Zhang M, Liu L, Lu S, Zhang CZ and Yun J: Decrease of fibulin-3 in hepatocellular carcinoma indicates poor prognosis. PLoS One 8: e70511, 2013.

25. Su PH, Lin YW, Huang RL, Liao YP, Lee HY, Wang HC, Chao TK, Chen CK, Chan MW, Chu TY, et al: Epigenetic silencing of PTPRR activates MAPK signaling, promotes metastasis and serves as a biomarker of invasive cervical cancer. Oncogene 32: 15-26, 2013.

26. Chen Q, Cao HZ and Zheng PS: LGR5 promotes the proliferation and tumor formation of cervical cancer cells through the Wnt/ $\beta$-catenin signaling pathway. Oncotarget 5: 9092-9105, 2014.

27. QI P and Du X: The long non-coding RNAs, a new cancer diagnostic and therapeutic gold mine. Mod Pathol 26: 155-165, 2013.

28. Qiu MT, Hu JW, Yin R and Xu L: Long noncoding RNA: An emerging paradigm of cancer research. Tumour Biol 34: 613-620, 2013.

29. Valle-Mendiola A, Weiss-Steider B, Rocha-Zavaleta L and Soto-Cruz I: IL-2 enhances cervical cancer cells proliferation and JAK3/STAT5 phosphorylation at low doses, while at high doses IL-2 has opposite effects. Cancer Invest 32: 115-125, 2014.

30. Xiang T, Du L, Pham P, Zhu B and Jiang S: Nelfinavir, an HIV protease inhibitor, induces apoptosis and cell cycle arrest in human cervical cancer cells via the ROS-dependent mitochondrial pathway. Cancer Lett 364: 79-88, 2015.

31. Pahne-Zeppenfeld J, Schröer N, Walch-Rückheim B, Oldak M, Gorter A, Hegde S and Smola S: Cervical cancer cell-derived interleukin-6 impairs CCR7-dependent migration of MMP-9-expressing dendritic cells. Int J Cancer 134: 2061-2073, 2014.

c) (7) $\odot$ This work is licensed under a Creative Commons cc) Attribution-NonCommercial-NoDerivatives 4.0 International (CC BY-NC-ND 4.0) License. 